Classification

Physics Abstracts

$68.55-68.15-75.10 \mathrm{H}$

\title{
Re-entrant wetting transitions in molecular fluid mixtures
}

\author{
T. J. Sluckin \\ Faculty of Mathematical Studies, University of Southampton, Southampton SO9 5NH, U.K. \\ and J. T. Chalker \\ Department of Physics, University of Southampton, Southampton SO9 5NH, U.K.
}

\begin{abstract}
Résumé. - L'adsorption préférentielle par un mur de molécules d'un mélange fluide est décrite par un modèle où on introduit des degrés de liberté orientationnels. L'énergie potentielle effective de l'interaction mur-molécule est alors fonction de la température. Dans des conditions appropriées, des transitions de mouillage ré-entrantes peuvent se produire. Lorsque la température décroît, la surface peut devenir successivement mouillée, puis sèche, puis mouillée et sèche à nouveau. Une transition de surface ré-entrante, analogue à la ligne de pré-mouillage de haute température, est associée aux phases mouillées. Des calculs exacts en dimension deux et l'approximation de champ moyen permettent de mettre en lumière ces phénomènes.
\end{abstract}

\begin{abstract}
Preferential adsorption of molecules in a molecular fluid by a wall is modelled by the introduction of orientational degrees of freedom, which cause the effective wall-molecule potential energy to be temperature dependent. Under suitable circumstances re-entrant wetting transitions can occur. As temperature is reduced the surface can be successively wet, dry, wet and dry again. Associated with the wet phase is a re-entrant surface phase transition analogous to the high temperature prewetting line. Exact two dimensional and approximate mean field calculations have been carried out to illuminate this phenomenon.
\end{abstract}

\section{Introduction.}

A binary liquid mixture of two components A and B, say, usually forms a single homogeneous phase at high temperatures but has a miscibility gap below a critical solution temperature. When the A-B mixture is placed in contact with a wall which has a preferential attraction for one type of molecule, there is in general a set of surface transitions associated with the first-order transition between the A-rich and B-rich phases. These wetting and drying transitions have been much studied recently [1-5]. The B-rich phase is said to wet the wall if, at coexistence between the A-rich and B-rich phases, a macroscopic layer of B-rich fluid intrudes between the A-rich fluid and the wall. The contact angle $\theta$ along a contact line between $A, B$ and wall in this situation is then zero. Partial wetting by the B-rich phase corresponds to a finite film thickness of B-rich fluid, or $0<\theta<\pi$. The structure of the surface phase diagram is rather general and is summarized in figure 1 . Near the critical temperature one phase (B, say) wets the walls. There may be a wetting temperature $T_{w}$ below which partial wetting occurs. Above $T_{w}$ there is a surface phase 


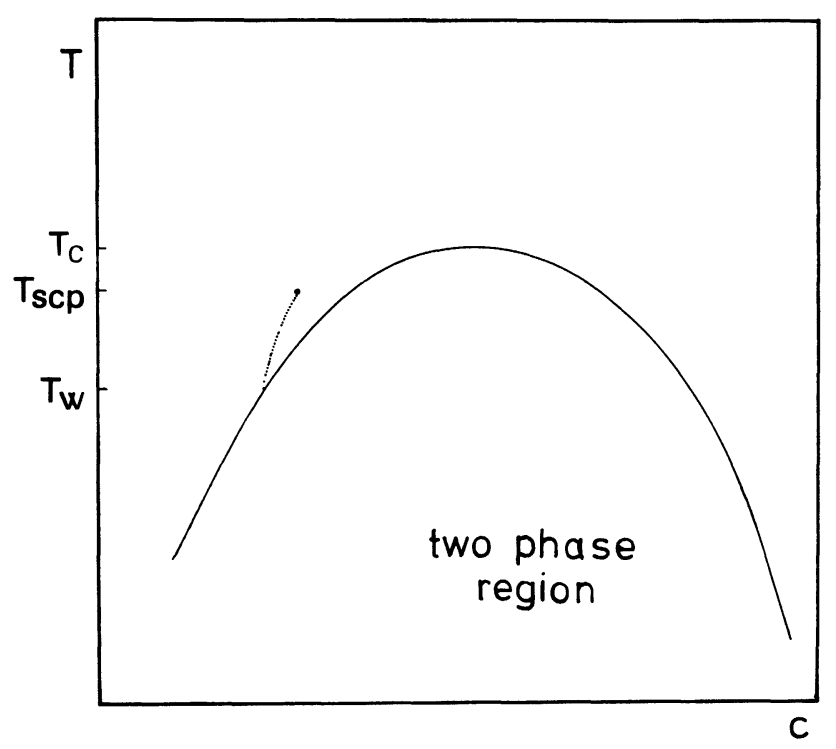

Fig. 1. - Schematic phase diagram for a simple A-B binary fluid mixture, with surface phase diagram superimposed. $c$ is the molar concentration of $\mathrm{B}$. Critical point is at $T_{\mathrm{c}}$, wetting temperature at $T_{\mathrm{w}}$. The prewetting line (dotted) joins the wetting transition to the surface critical point at $T_{\text {scp }}$.

transition in the A-rich phase, near the first-order bulk transition, corresponding to a discontinuous change in the thickness of the adsorbed B-rich film at the wall. The locus of this transition in the phase diagram - the so-called prewetting line - starts at $T_{\mathrm{w}}$ on the bulk coexistence line and ends at a surface critical point. The order of the surface phase transition is intimately connected with the existence of the prewetting line - a first order wetting imposes a prewetting line, whereas a second order transition forbids it.

The simple bulk phase diagram shown in figure 1 is a consequence of the competition between the energetics of interparticle interaction and the entropy of mixing. The properties of more complex mixtures are affected by configurational entropic considerations. In particular the phase diagram may develop [6] necks (Fig. 2a) or even closed loops (Fig. 2b). Such fluid mixtures usually involve molecules with highly directionally dependent interactions; a recent theoretical discussion is given by Goldstein and Walker [7], and by Walker and Vause [8]. It is of some interest to ask how these exotic bulk phase diagrams will affect the wetting behaviour along the A-B miscibility line. Some speculations in answer to this are shown in figures 2 . It seems likely that the bulk re-entrant behaviour will enforce surface re-entrant behaviour as well. The perfect wetting near the critical point in figure 1 is merely a consequence of the rapid decrease in the A-B surface tension [3] as the critical point is approached; probably as a lower critical point is approached the same considerations will apply (Fig. 2b), enforcing perfect wetting here too. There may nevertheless be an intermediate region of partial wetting. Furthermore, a narrow neck in the phase diagram (Fig. 2a) may be enough to lower the A-B surface tension sufficiently to impose perfect wetting in the region of the neck. Associated with the first-order wetting transitions will be surface phase transitions - low temperature prewetting lines away from coexistence. Indeed, if the A-A, A-B and B-B potentials are changed smoothly in such a way that the phase diagram in figure 1 is changed smoothly into that of figure $2 a$ and then figure $2 b$, we expect the following. First, the lower prewetting line emerges and then later, as the closed loop in figure $2 \mathrm{~b}$ breaks away, the lower prewetting line splits into two at a surface bi-critical point. 
(a)

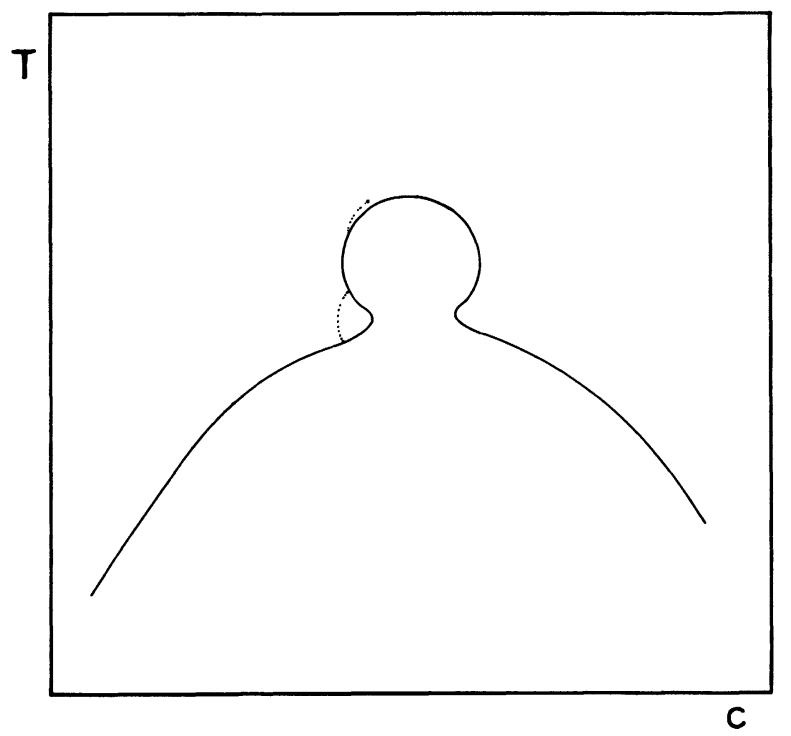

(b)

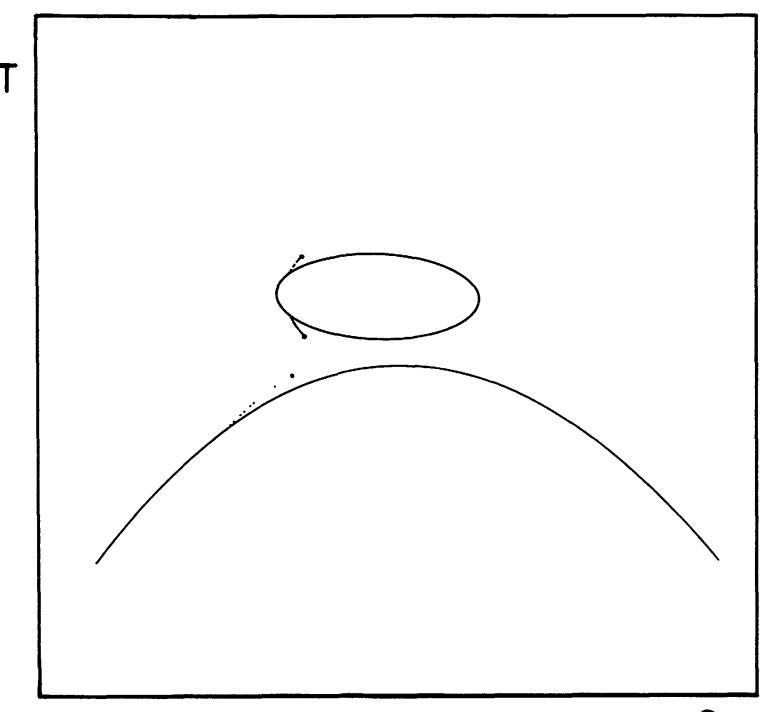

C

Fig. 2. - (a) A neck has appeared in the phase diagram, and a lower prewetting line in the surface phase diagram. (b) The neck has broken, separating an upper loop with a lower and upper critical point. There are a number of wetting transitions and associated prewetting lines.

In this Letter we study the question of whether re-entrant wetting behaviour can occur even if no exotic bulk phase behaviour is driving it. In our models the wall-molecule interactions are strongly orientationally dependent, whereas the inter-molecular interaction are taken to be orientationally independent. In fact, of course, if A and B consist of markedly anisotropic molecules, we may expect that both effects will occur; nevertheless it is desirable from a theoretical point of view (and simple from a computational point of view) to separate surface-driven from bulk-driven effects. We study two very idealized models : an exactly solvable two dimensional semi-infinite Ising lattice gas, and a mean field model for the free energy density of a two compo- 
nent fluid near an interface. In both cases, following Goldstein and Walker [7], we use a Potts model to mimic orientationally dependent forces. The bulk phase diagram is conventional. Nevertheless, in both cases, for suitable values of the model parameters, we discover more than one wetting transition. Two-dimensional wetting transitions are second order [8], and there are no associated prewetting lines. In the mean field theory a rich structure of lower prewetting lines is found, associated with the low temperature wet regime.

We now discuss our models in somewhat greater detail.

\section{Two-dimensional model.}

The model we study is defined as follows. Every site $i$ of a semi-infinite square lattice is occupied by a molecule which may be of type A or B; the variable $\sigma_{i}$ takes the values +1 or -1 accordingly. In addition, the molecules have orientational degrees of freedom which are represented by allowing each $\mathrm{A}$ (B) molecule to be in one of $q_{\mathrm{A}}\left(q_{\mathrm{B}}\right)$ orientational states; the variable $q_{i}$ takes the values $1,2, \ldots, q_{\mathrm{A}}$ when the site $i$ is occupied by an A molecule, and the values $1,2, \ldots, q_{\mathrm{B}}$ otherwise. Molecules at adjacent sites interact with an energy that depends only on their type, not on their orientation. The edge of the semi-infinite lattice represents a wall in contact with the fluid. There is an energetic preference for one species (say A) to occupy sites next to the wall, and one orientation - «bonding" - is favoured over others. Finally, there is a chemical potential for each species. The configurational energy is

$$
\begin{aligned}
J= & -J \sum_{\langle i j\rangle} \sigma_{i} \sigma_{j}-H_{0} \sum_{i}^{\prime} \sigma_{i}-\left(\mu_{\mathrm{A}}-\mu_{\mathrm{B}}\right) \sum_{i} \sigma_{i}- \\
& -\frac{H_{\mathrm{A}}}{\left(q_{\mathrm{A}}-1\right)} \sum_{i}^{\prime} \delta_{\sigma_{i+}}\left\{q_{\mathrm{A}} \delta_{q_{i} 1}-1\right\}- \\
& -\frac{H_{\mathrm{B}}}{\left(q_{\mathrm{B}}-1\right)} \sum_{i}^{\prime} \delta_{\sigma_{i-}}\left\{q_{\mathrm{B}} \delta_{q_{i} 1}-1\right\},
\end{aligned}
$$

where $\sum_{\langle i j\rangle}$ is a sum over nearest-neighbour pairs, $\sum_{i}^{\prime}$ is a sum over edge sites and $\sum_{i}$ is a sum over all sites $[7,8]$.

The trace over the $\left\{q_{i}\right\}$ in the partition function is straightforward to calculate since we have neglected any coupling between orientations on different sites. In this way an effective, temperature dependent, Hamiltonian for the occupation variables is obtained :

$$
\mathcal{H}_{\mathrm{eff}}=-J \sum_{\langle i j\rangle} \sigma_{i} \sigma_{j}-H_{\mathrm{eff}} \sum_{i}^{\prime} \sigma_{i}-\mu_{\mathrm{eff}} \sum_{i} \sigma_{i}+\text { constant },
$$

where

$$
\begin{aligned}
& H_{\text {eff }}=H_{0}+\frac{1}{2} \beta^{-1}\left\{\ln \left[\frac{1}{q_{\mathrm{A}}}\left(\mathrm{e}^{\beta H_{\mathrm{A}}}+\left(q_{\mathrm{A}}-1\right) \mathrm{e}^{-\beta H_{\mathrm{A}} /\left(q_{\mathrm{A}}-1\right)}\right)\right]-\right. \\
& \mu_{\text {eff }}=\mu_{\mathrm{A}}-\mu_{\mathrm{B}}+\frac{1}{2} \beta^{-1} \ln \left(q_{\mathrm{A}} / q_{\mathrm{B}}\right)
\end{aligned}
$$

with $\beta$ the inverse temperature.

Exact results concerning surface wetting in this model at coexistence $\left(\mu_{\text {eff }}=0\right)$ have been derived by Abraham [9]. Below the bulk critical temperature, $T_{c}$, there are two phases : an A-rich and a B-rich. If the bulk of the system is in the B-phase and $H_{\text {eff }}$ is sufficiently positive, there will 
be an adsorbed layer of the A phase at the surface. The thickness of the adsorbed layer diverges as the wetting temperature, $T_{w}$, is approached from below and remains infinite for temperatures between $T_{\mathrm{w}}$ and $T_{\mathrm{c}}$. The wetting temperature was found by Abraham [9] to be the non-trivial solution of

$$
\mathrm{e}^{2 \beta J}\left[\cosh 2 \beta J-\cosh 2 \beta H_{\text {eff }}\right]=\sinh 2 \beta J .
$$

In our model the coupling between molecular orientations and the surface results in a temperature dependent $H_{\text {eff }}$ and hence (for appropriate parameter values) re-entrant surface wetting. A representative slice of the full (six-dimensional) phase diagram is shown in figure 3. For various choices of the parameters the surface may be successively wet; dry-wet; dry-wet-dry-wet or wet-dry-wet, as temperature is increased from zero to $T_{\mathrm{c}}$.

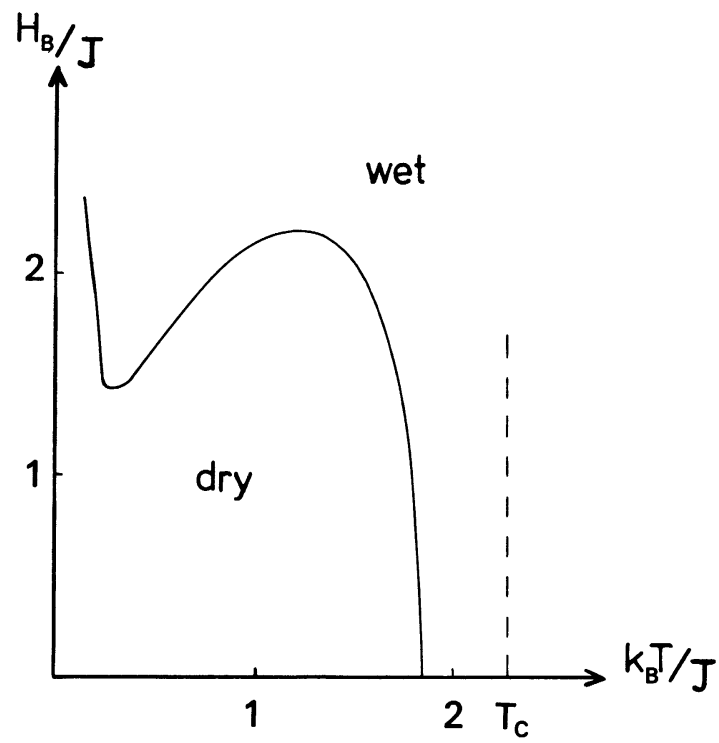

Fig. 3. - Surface phase diagram for the two-dimensional model described in the text. The parameter values chosen were $J=1, H_{0}=0.6, q_{\mathrm{A}}=10, q_{\mathrm{B}}=100, H_{\mathrm{A}}=1.2 H_{\mathrm{B}}$.

\section{Mean field theory.}

We adopt a bulk free energy density, $F(S)$, of the Ginzburg-Landau form, with an order parameter $S$, and

$$
\begin{aligned}
& F(S)=f(S)+\frac{1}{2} K\left(\frac{\mathrm{d} S}{\mathrm{~d} z}\right)^{2} \\
& f(S)=a\left(T-T_{\mathrm{c}}\right) S^{2}+b S^{4}-V S
\end{aligned}
$$

$V$ is an external field, and $V \neq 0$ corresponds to being away from coexistence. We assume for simplicity, that $a, b, K$ are temperature independent. The spontaneous bulk order parameter $S(\infty)$ is given by

$$
S(\infty)= \pm S_{0}(1-t)^{1 / 2}
$$

where $t=T / T_{\mathrm{c}}$.

We suppose that the bulk B phase at $z=\infty$ has $S$ negative. 
The surface free energy per unit area is of the Cahn form :

$$
\sigma=F_{0}\left(S_{\mathrm{s}}\right)+\int_{0}^{\infty}\left[f(S(z))-f(S(\infty))+\frac{1}{2} K\left(\frac{\mathrm{d} S}{\mathrm{~d} z}\right)^{2}\right] \mathrm{d} z
$$

where $S_{\mathrm{s}}=S(z=0)$ is the value of $S$ at the wall, taken to be at $z=0$ in the $x-y$ plane.

As in section 2 the molecules in the surface may be in specific orientational states $-q_{\mathrm{A}}$ for A molecules and $q_{\mathrm{B}}$ for B molecules. The only effect of the Potts degree of freedom is to cause the effective surface field to be temperature dependent. Then

$$
F_{0}\left(S_{\mathrm{s}}\right)=-H_{\mathrm{eff}}(T) S_{\mathrm{s}}
$$

where $H_{\text {eff }}$ is given in equation (2.2).

Solving the Euler-Lagrange equations for the functional (3.3) one obtains

$$
\begin{gathered}
\sigma=F_{0}\left(S_{\mathrm{s}}\right)+\sqrt{2 K} \int_{S_{\mathrm{s}}}^{S(\infty)}[f(S)-f(S(\infty))]^{1 / 2} \mathrm{~d} S \\
\frac{1}{(2 K)^{1 / 2}} \frac{\mathrm{d} F_{0}}{\mathrm{~d} S}\left(S_{\mathrm{s}}\right)=[f(S)-f(S(\infty))]^{1 / 2} .
\end{gathered}
$$

Equation (3.6) may have one solution for $S_{\mathrm{s}}>+|S(\infty)|$, or it may have two (stable) solutions, one for $S_{\mathrm{s}}>+|S(\infty)|$ and the other for $S_{\mathrm{s}}<+|S(\infty)|$. The B phase wets the surface if the large $S_{\mathrm{s}}$ solution gives lowest free energy in equation (3.5). The regions of stability are decided by the dimensionless quantity

$$
\gamma(T)=\frac{H_{\mathrm{eff}}(T)}{(2 K b)^{1 / 2} S(\infty)^{2}} .
$$

The following results can be easily calculated :

(i) $\gamma<\gamma_{1}:$ Dry.

(ii) $\gamma_{1}<\gamma<\gamma_{2}$ : Wet. However at coexistence there exists a metastable dry value of $S_{\mathrm{s}}$.

(iii) $\gamma_{2}<\gamma<\gamma_{3}$ : Wet. At coexistence there is no metastable dry value of $S_{\mathrm{s}}$. In this regime, and in regime (ii) however, there exists a thin film - thick film transition away from coexistence.

(iv) $\gamma_{3}<\gamma$ : Wet. There is no surface phase transition. For our present model : $\gamma_{1}=0.68$, $\gamma_{2}=1$ and $\gamma_{3}=\sqrt{3}$.

We can re-write $\gamma(T)$ in terms of dimensionless quantities

$$
\begin{aligned}
& \gamma_{0}=\frac{H_{0}}{(2 K b)^{1 / 2} S_{0}^{2}} \\
& \lambda=H_{\mathrm{A}} / 2 H_{0} \\
& \mu=H_{\mathrm{B}} / 2 H_{0} \\
& x=2 H_{0} / T_{\mathrm{c}} \\
& \gamma(t)=\gamma_{0}\left[1+\frac{t}{x} \ln \left\{\frac{1}{q_{\mathrm{A}}}\left(\mathrm{e}^{x \lambda / t}+\left(q_{\mathrm{A}}-1\right) \mathrm{e}^{-x \lambda / t\left(q_{\mathrm{A}}-1\right)}\right)\right\}-\right. \\
&-\frac{t}{x} \ln \left\{\frac{1}{q_{\mathrm{B}}}\left(\mathrm{e}^{-x \mu / t}+\left(q_{\mathrm{B}}-1\right) \mathrm{e}^{-x \mu / t\left(q_{\mathrm{B}}-1\right)}\right)\right\} .
\end{aligned}
$$


The quantity $\gamma(t)$ may have complicated behaviour, due to competition between the factor $(1-t)^{-1}$ which steadily increases it, and the logarithmic terms which decrease at higher temperatures when the molecules at the surface are no longer oriented. There are consequently several possibilities for the surface phase diagram, some of which are exhibited in figure 4 .

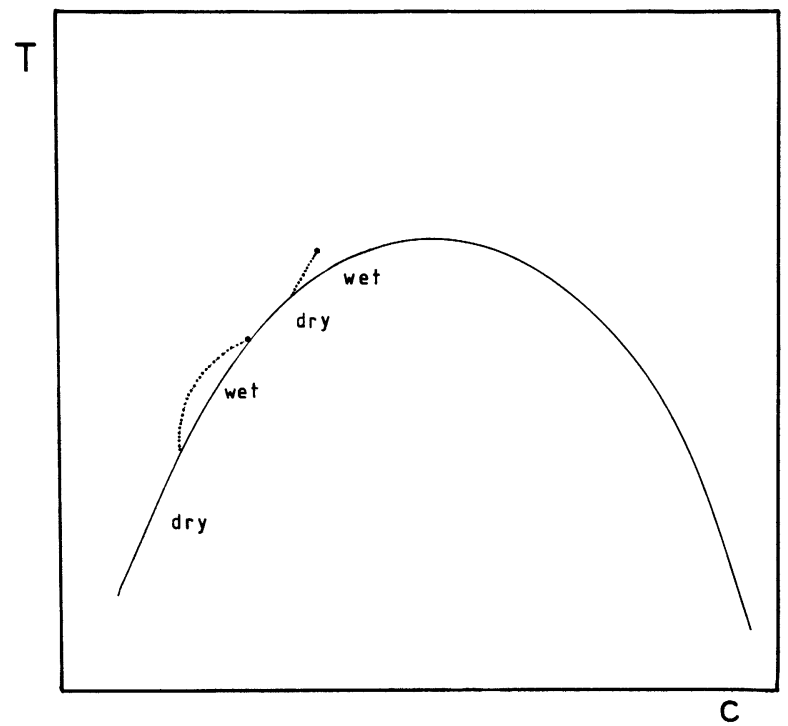

Fig. 4a. - Surface phase diagram (schematic) with reentrant wet and dry phases, and continuous lower prewetting line.

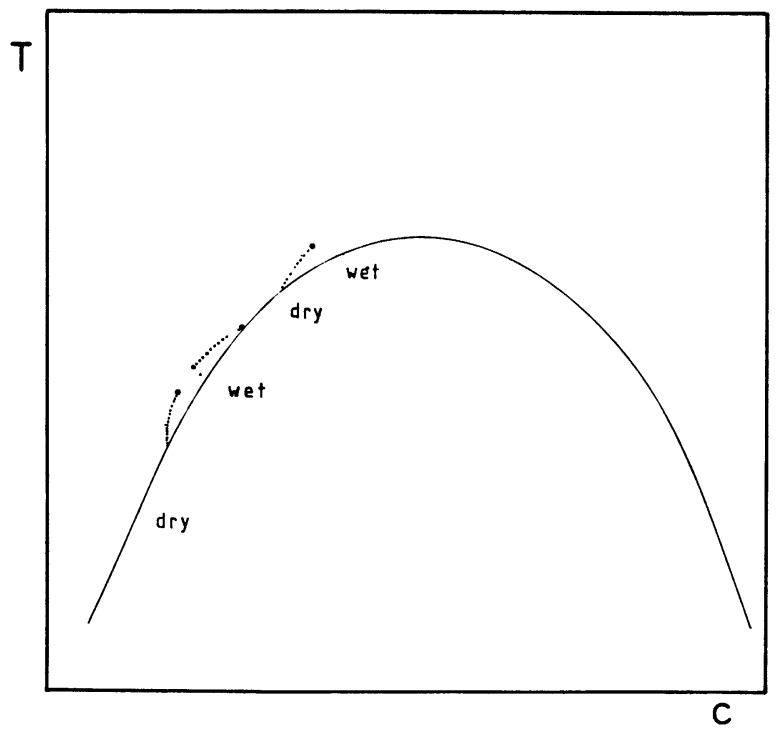

Fig. 4b. - Surface phase diagram with reentrant wet and dry phases, and discontinuous lower prewetting lines. 


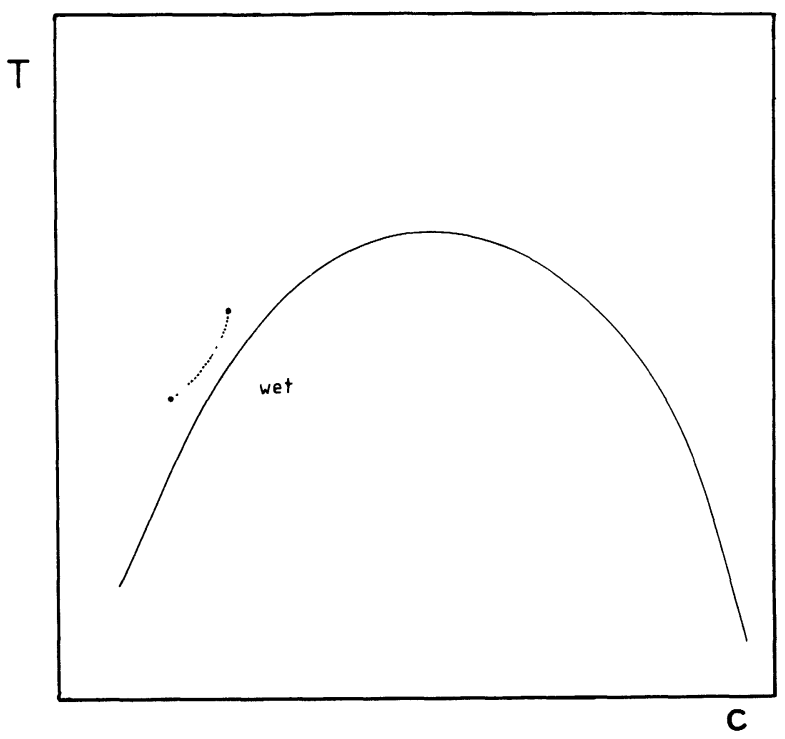

Fig. 4c. - Surface phase diagram for totally wet surface with disconnected prewetting line.

The simple phase diagram (Fig. 1) has a dry phase at low $t$ and a wet phase for $t$ near 1 . In figures $4 \mathrm{a}$ and $4 \mathrm{~b}$, as temperature is decreased, the surface is successively wet, dry, then a re-entrant wet phase, and finally dry again. The very low temperature dry phase is a consequence of selective binding of A molecules to the surface; in the absence of this phenomenon, the low temperature phase is wet. In any case, there are low temperature prewetting lines associated with each wetting transition. In figure 4a the line associated with the transition between the low temperature wet phase and the neighbouring dry phases is continuous - there is no surface critical point associated with this line. There is of course still a prewetting line and a surface critical point associated with the high temperature wet phase. In figure $4 \mathrm{~b}$ the phase diagram at coexistence is as in figure $4 \mathrm{a}$, but the continuous prewetting line has split into two parts each of which ends in a surface critical point. The two surface critical points emerge at a bicritical point corresponding to $\gamma(t)=\gamma_{3}, \frac{\mathrm{d} \gamma}{\mathrm{d} t}(t)=0$.

In figure $4 \mathrm{c}$ both the low temperature and high temperature surfaces are wet, the one on energetic and the other on entropic grounds. There is no intermediate dry phase, but the attempt to produce one does leave a ghost - a prewetting line away from coexistence all the way along its length, at each end of which is a surface critical point. As parameters are changed the middle of the prewetting curve meets the coexistence curve at a critical point, beyond which there are two first order transitions at coexistence and a dry phase in between.

Finally, in figure 5 we show the surface phase diagram as a function of $t$ and $\lambda$, for fixed $\gamma$, $x, \mu / \lambda$. This is the analogue of figure 3 , for the mean field model. As in figure 3 the wet and dry regions of parameter space are each connected, but there is a region of intermediate $\lambda$ for which the curve is reentrant.

\section{Conclusions.}

We have raised the possibility that the surface wetting transition may under some circumstances be re-entrant, suggesting two mechanisms that might be responsible : incipient bulk re-entrancy, 


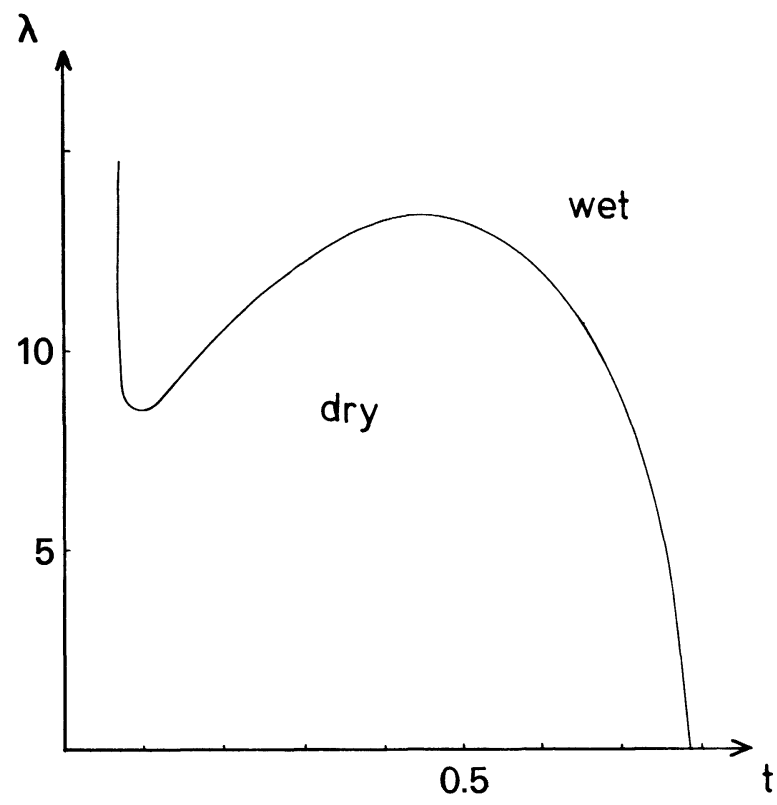

Fig. 5. - Surface phase diagram as a function of $\lambda$ and $t$, with $\mu / \lambda=0.8, \gamma_{0}=0.15, x=0.05, q_{\mathrm{A}}=10$, $q_{\mathrm{B}}=100$.

and orientationally dependent interactions between fluid molecules and the surface. The second has been analysed in some detail.

Both these mechanisms are most likely to operate in binary mixtures consisting of highly anisotropic molecules. It is known [10] that under some circumstances there can be strong orientational effects in the absorption of molecules on surfaces. We may ask from a physical point of view what is driving the various wetting and drying transitions. For a simple fluid mixture of components $\mathrm{A}$ and $\mathrm{B}$, the component $\mathrm{A}$ may wet the wall near the critical point - this corresponds to entropic behaviour dominating - while at lower temperatures there will only be partial wetting, corresponding to energy effects winning. For mixtures of fluids of complicated molecules it may well be that at the critical point the wall only sees the orientationally averaged interaction with molecules A or B. However as the temperature is lowered the crucial feature will not be the average interaction, but the interaction with the most favoured orientation. At low temperatures this may favour the wall being wet by the A phase or it may not, depending on how strong are the wall-molecule interactions. The re-entrant wet phase will occur, if it does so, when the strong A-wall interactions are "turned on » by a reduced temperature, but strong B-wall interactions have not been turned on. If the $A(B)$-wall interactions are characterized by parameters $J_{A}\left(J_{B}\right)$, then the strongly orientational interactions are "turned on " at temperatures $T \approx J / K_{\mathrm{B}} \ln q$, where $q$ is the multiplicity of the orientational degrees of freedom. Thus if $J_{\mathbf{B}}>J_{\mathrm{A}}$ we expect that the A phase will not wet at low temperatures, but we may still get a low temperature wet phase if

$$
\bar{T}_{\mathrm{w}}>J_{\mathrm{B}} / \ln q_{\mathrm{B}},
$$

if the A molecule has a lower number of orientational states, and $\bar{T}_{\mathrm{w}}$ is the wetting temperature determined for spherically averaged molecules. The quantity $q$ is a model parameter, but it clearly is related to the width of the distribution of favoured orientations in the space of all possible orientations. 
Finally we note that re-entrant wetting has been discussed in a different context by Seguin $e t$ al. [11] and Bienfait et al. [12]. These authors are not discussing the phenomenon that we consider. Their experiments concern low temperature wetting of surfaces by rare gas solids. They compare adsorption of different rare gas solids, which should be characterized, according to contemporary theory, by a monotonically increasing parameter, driving (as the system is changed) the surface phases from wet to dry. They discover anomalous behaviour, which has been very recently interpreted in terms of solid state strain effects [13]. In contrast we are considering wetting of surfaces at liquid-liquid equilibrium and allowing temperature to change monotonically. We do not expect that these effects will exhibit themselves in, for instance, argon-methane mixtures, and it seems unlikely that they will be important in discussing wetting at liquid-vapour equilibrium.

\section{References}

[1] DASH, J.G.,Phys. Rev. B 15 (1977) 3136.

[2] Sullivan, D.E., Phys. Rev. B 20 (1979) 3990.

[3] CahN, J.W., J. Chem. Phys. 66 (1977) 3667.

[4] Pandit, R., Schick, M. and Wortis, M., Phys. Rev. B 26 (1982) 5112.

[5] CoHN, R.H. and JACOBS, D.T., J. Chem. Phys. 80 (1984) 856 ; (These authors find non-wetting behaviour at a point, contrary to other theoretical and experimental indications. This curious anomalous behaviour clearly merits further investigation and is inconsistent with our theoretical picture).

[6] van Konynenberg, P.H. and Scott, R.L., Philos. Trans. R. Soc. A 298 (1980) 495.

[7] Goldstein, R.E. and Walker, J.S., J. Chem. Phys. 78 (1983) 1492.

[8] Walker, J.S. and Vause, C.A., J. Chem. Phys. 79 (1983) 2660.

[9] Aвraham, D.B., Phys. Rev. Lett. 44 (1980) 1165.

[10] Adamson, A.W., Physical Chemistry of Surfaces (Wiley) 1976.

[11] Seguin, J.L., Suzanne, J., Bienfait, M., Dash, J.G. and Venables, J.A., Phys. Rev. Lett. 51 (1983) 122.

[12] Bienfait, M., Seguin, J.L., Suzanne, J., Lerner, E. and Krim, J., Phys. Rev. B 29 (1984) 983.

[13] Huse, D.A., GitTes and SCHICK, M., Preprints 1984. 